\title{
Showing why Measures of Quantified Beliefs are Belief Functions
}

\author{
Philippe Smets \\ IRIDIA \\ Université Libre de Bruxelles \\ 50 av. Roosevelt, CP 194-6, 1050 Bruxelles, Belgium \\ psmets@ulb.ac.be
}

\begin{abstract}
In $[8,10]$, we present an axiomatic justification for the fact that quantified beliefs should be represented by belief functions. We show that the mathematical function that can represent quantified beliefs should be a Choquet capacity monotone of order 2. In order to show that it must be monotone of order infinite, thus a belief function, we propose several extra rationality requirements. One of them is based on the negation of a belief function, a concept introduced by Dubois and Prade [2]. This concept was essentially abstract, and its applicability was neither established nor illustrated. Here we present an illustrative example of this negation process. This example gives ground to the use of belief functions to represent quantified beliefs.
\end{abstract}

Keywords: TBM, belief function, belief representation

\section{Introduction}

The use of any mathematical model to represent quantified beliefs, i.e., weighted opinions, can be supported either by defending convincing definitions with illustrative examples or by producing a set of axioms that justify it. For what concerns the models based on belief functions, examples illustrating the first approach can be found among others in $[7,4,12,11]$ whereas the second approach is developed in $[15,8,10])$.

In any model for quantified beliefs, one considers an agent, the belief holder, called You hereafter, and a finite frame of discernment, denoted $\Omega$. One of the worlds in $\Omega$, denoted $\omega_{0}$, is the actual world, but, due to Your limited understanding, You cannot state which world is the actual one. All You can express is the strength of Your opinions, called hereafter beliefs, that $\omega_{0}$ belongs to $A$ for every $A \subseteq \Omega$. We assume that this belief is represented by a pointwise measure defined on $2^{\Omega}$, the power set of $\Omega$.

This measure is temporarily denoted by $\mathrm{Cr}$ and called a 'credibility function'. So for every $A \subseteq \Omega, \operatorname{Cr}(A)$ expresses (the strength of) Your belief that the actual world $\omega_{0}$ belongs to $\mathrm{A}$.

In $[8,10]$, we produce sets of rationality requirements that should be satisfied by any credibility function and we prove 1) belief functions satisfy them, 2) probability functions, 
that are special cases of belief functions, are insufficiently expressive to represent degrees of belief and 3) Choquet capacities [1], that are the generalization of belief functions, violate some of the requirements.

During the demonstration, we produce requirements from which we prove 1) the convexity of the set of credibility functions, 2) how credibility functions are adapted by uninformative modifications of the frame of discernment (refinement and coarsening), and 3 ) how they are revised by conditioning. At that level, we prove that $\mathrm{Cr}$ is a Choquet capacity monotone of order 2 [1]. To show that it is monotone of infinite order (i.e., a belief function), we propose several extra requirements. One of them is based on the negation of a belief function, a concept invented by Dubois and Prade [2]. It states: 'the negation of a credibility function is a credibility function'. In that case credibility functions are belief functions.

Unfortunately, this negation concept was only a mathematical property. To be used as a rationality requirement, one must produce at least one practical illustrative and convincing example where the negation is used.

In this paper, we present such an example. Thanks to it, our axiomatic justification presented in $[8,10]$, is simplified.

\section{Credibility functions}

Let $C r$ represent Your belief over $\Omega$, a finite frame of discernment. The only properties of $\mathrm{Cr}$ used in this paper are:

1. Bounded non negativity: $\operatorname{Cr}: 2^{\Omega} \rightarrow[0,1]$ where $\operatorname{Cr}(\emptyset)=0$ and $\operatorname{Cr}(\Omega) \leq 1$

2. Monotony to inclusion: $\forall A, B \subseteq \Omega$, if $A \subseteq B$, then $\operatorname{Cr}(A) \leq C r(B)$

3. Revision: the revision of $C r$ by a piece of evidence $E v$ is represented by a $2^{|\Omega|} \times$ $2^{|\Omega|}$ matrix $H^{*}$ which depends on $\mathrm{Ev}$ but not on $\mathrm{Cr}$, and the revised credibility function $C r[E v]$ is given by the matricial product $C r[E v]=H^{*} \cdot C r$.

The first requirement is quite strong as it eliminates models based on sets of probability functions $[5,6,13]$ or on interval valued probabilities [14]. We accept the closed world assumption $(\operatorname{Cr}(\Omega)=1)$ in order to avoid useless discussions. The second requirement is assumed by any model of uncertainty and hardly questionable. The third requirement translates into the belief function framework the transformation achieved by a Markow matrix in classical probability theory. It satisfies quite natural requirements, and could almost be just assumed, what we do here. Proving that it is a necessary property will be presented in a forthcoming paper. That $H^{*}$ is a stochastic matrix can be deduced when $\mathrm{Cr}$ is a belief function. But as far as the purpose of this paper is to prove that $\mathrm{Cr}$ is a belief function, we can neither assume nor deduce it.

We present some needed background material (see $[12,11])$.

\subsection{Notation convention}

$C r^{\Omega}[E v](B)$ is the degree of belief given by You that the actual world belongs to $B$, which is a subset of $\Omega, E v$ is a set of propositions (called the Evidential Corpus) and You accept as true the propositions deduced from those in $E v$ ( $E v$ covers the classical conditioning event). The domain will be omitted when no confusion can occur. 
The term between [ and ] is what You accept as true. In particular, it can be the conditioning event encountered in probability theory. Note that Your beliefs are based on what You accept as true, not on what is true. There is no necessity that what You accept as true is true, it might perfectly be false. Your beliefs would be 'unjustified', 'inadequate', 'erroneous', but so it is. Rationality is trying to accept only what is true, but this is only an ideal goal, and daily reality is far from that ideal.

By convention when we write $C r^{\Omega}[\omega]$ for $\omega \in \Omega$, we mean that You accept as true that $\omega_{0} \in \omega$ and do not accept as true that $\omega_{0} \in \omega^{*}$ for any $\omega^{*} \subset \omega$ (where $\subset$ denotes strict subset). We will say that $\omega$ is 'all You accept as true'.

A credibility function can itself be part of the evidential corpus. For example, we write $C r^{\Omega}\left[\omega, C r^{\Theta}\right]$ to mean that You accept as true both that $\omega_{0} \in \omega$ and that Your beliefs about $\Theta$ is represented by $C r^{\Theta}$.

\subsection{Doxastic equivalence and consistency}

The next definition translates the idea that if two propositions are equivalent given what You know, the credibility functions they induce are equal.

Definition 2.1 Doxastic equivalence. Let Ev be an 'Evidential Corpus', that is a set of propositions that You accept as true. Two propositions $p_{1}$ and $p_{2}$ are said to be doxastically equivalent under an Evidential Corpus Ev, what is denoted by $p_{1} \equiv_{E v} p_{2}$, when $p_{1} \wedge$ Ev and $p_{2} \wedge$ Ev are logically equivalent.

We then assume:

Proposition 2.1 Doxastic consistency. If $p_{1} \equiv_{E v} p_{2}$, then

$$
C r^{\Omega}[E v]\left(p_{1}\right)=C r^{\Omega}[E v]\left(p_{2}\right) .
$$

\subsection{The Möbius transform}

Let $C r^{\Omega}$ be a credibility function defined on $\Omega$, its Möbius transform, denoted $m^{\Omega}$ is defined as:

$$
m^{\Omega}(A)=\sum_{B \subseteq A}(-1)^{|A|-|B|} C r^{\Omega}(B), \forall A \subseteq \Omega
$$

We call $m^{\Omega}$ the Möbius mass function and $m(A)$ a Möbius mass. In particular, the basic belief assignment is the Möbius transform of a belief function. $\mathrm{Cr}^{\Omega}$ is a belief function iff $m^{\Omega}(A) \geq 0$ for all $A \subseteq \Omega$ and $\sum_{A \subseteq \Omega} m^{\Omega}(A)=1$.

\subsection{Coarsening}

Let $\Omega$ and $\Omega^{*}$ be two frame of discernments where the elements of $\Omega^{*}$ are the elements of a partition of $\Omega$. For $B \subseteq \Omega$, let $\operatorname{Coars}(B)$ denote the smallest subset of $\Omega^{*}$ that contains $B$. We call $\Omega^{*}$ an uninformative (it means 'just redefining the frame') coarsening of $\Omega$. Given $C r^{\Omega}$ on $\Omega, C r^{\Omega^{*}}(A)=C r^{\Omega}(A)$ for all $A \subseteq \Omega^{*}$ (if $A \subseteq \Omega^{*}$, then $A \subseteq \Omega$ ). It results from the doxastic consistency requirement. In that case,

$$
\forall A \subseteq \Omega^{*}, m^{\Omega^{*}}(A)=\sum_{B \subseteq \Omega, \operatorname{Coars}(B)=A} m^{\Omega}(B) .
$$




\subsection{All You accept as true}

Let the frame of discernment $\Omega$, and suppose all You accept as true is that $\omega_{0} \in \omega$ for an $\omega \subseteq \Omega$. What is the credibility function $\mathrm{Cr}^{\Omega}[\omega]$ induced on $\Omega$ under that condition? Requiring $C r^{\Omega}[\omega](\omega)=1$ and $C r^{\Omega}[\omega](\bar{\omega})=0$ seem natural.

What about You beliefs about $\omega^{*} \subset \omega$ ? It seems also natural to require that all strict subsets $\omega^{*}$ of $\omega$ receive the same belief. For instance, why should any of them be better supported that it complement relative to $\omega$ ? The concept of cardinality of the set $\omega$ cannot be used, as beliefs would otherwise violate the doxastic consistency requirement (see [10]). Let $\beta$ be that particular value. The term $\beta$ cannot be negative as it is among others the belief given to the singletons of $\omega$. If furthermore we require that beliefs can never be smaller than $\beta$, then $\beta=0$ as for $\bar{\omega}, C r^{\Omega}[\omega](\bar{\omega})=0$.

Finally, the beliefs given to any $\omega^{*}$ is equal to the beliefs given to $\omega^{*} \cap \omega$, as the worlds in $\omega^{*}$ but not in $\omega^{*} \cap \omega$ belong to $\bar{\omega}$ and are thus accepted by You as impossible given You accept $\omega$ as true.

The next proposition express these ideas:

Proposition 2.2 The credibility function that represents Your beliefs given all You accept as true is $\omega_{0} \in \omega$ is given by:

$$
\begin{aligned}
{[2] C r^{\Omega}[\omega]\left(\omega^{*}\right) } & =1 & & \text { if } \omega \subseteq \omega^{*} \\
& =0 & & \text { if } \omega^{*} \subset \omega \\
& =C r^{\Omega}[\omega]\left(\omega^{*} \cap \omega\right) & & \text { otherwise }
\end{aligned}
$$

The Möbius transform of $C r^{\Omega}[\omega]$ is given by:

$$
\begin{aligned}
{[2] m^{\Omega}[\omega]\left(\omega^{*}\right) } & =1 & & \text { if } \omega=\omega^{*} \\
& =0 & & \text { otherwise }
\end{aligned}
$$

\subsection{Revision}

The next theorem reexpresses the $\mathrm{Cr}$ revision requirement under an equivalent but more convenient form. We had assumed that the revision of a credibility function $\mathrm{Cr}$ by a piece of evidence $E v$ can be represented by a matrix $H^{*}$ such that the revised credibility function $C r[E v]$ is equal to $H^{*} \cdot C r$.

Theorem 2.1 Let $C r$ and $C r^{*}$ be two credibility functions defined on $\Omega$. If $C r^{*}=H^{*}$. $C r$, there exists a $2^{|\Omega|} \times 2^{|\Omega|}$ matrix $H$ such that $C r^{*}=H \cdot m$ where $m$ is the Möbius transforms of $\mathrm{Cr}$. In that case,

$$
C r^{*}(A)=\sum_{B \subseteq \Omega} h(A, B) m(B), \forall A \subseteq \Omega
$$

where $h(A, B)$ are the elements of the matrix $H$.

Proof. Let $M$ be the operator (a matrix) that transforms any credibility function on $\Omega$ into its Möbius transforms [11]. $M$ is not singular, so $M^{-1}$ exists. $C r^{*}=H^{*} \cdot C r$ can be rewritten as $C r^{*}=H^{*} \cdot M^{-1} \cdot M \cdot C r$, thus $C r^{*}=H \cdot m$ where $H=H^{*} \cdot M^{-1}$. The equation is just a rewriting of the matricial equality. 
In order to prove that $\mathrm{Cr}$ is a belief function, we must produce an example that shows that if some of the values of $m$ are negative, there exists a $H$ matrix such that some values of $\mathrm{Cr}^{*}$ are negative. To produce such a matrix is mathematically trivial, but the challenge was to find a practical example that leads to such a matrix. This is what we achieve in the next section.

\section{The Mischievous Killer}

We consider here only the proof that the Möbius mass given to the $\Omega$ when $|\Omega|=3$ may not be negative. The case with 2 was proved in [10]. The real challenge was to go from 2 to 3 . So we consider that case first.

Our example is based on a murder scenario, but could as well be rephrased as any diagnostic - detection problem.

\subsection{The scenario}

Suppose a murder has been committed by a single killer, denoted $k_{0}$, and there are three suspects named $A, B$, and $C$. We denote by $D$ 'anybody else'. So You know for sure that the killer $k_{0} \in \Omega$ with $\Omega=\{A, B, C, D\}$.

You collect a piece of evidence, a cigarette butt which brand, denoted $\theta_{0}$. The domain for $\theta_{0}$ is $\Theta=\{a, b, c, d\}$. The butt you observe can only be one of $\{a, b, c\}$. You look at the butt and build a belief $C r^{\Theta}$ about the actual value $\theta_{0}$.

The $C B$ piece of evidence. You know the next piece of evidence, denoted $C B$ for cigarette butt,

1. $\theta_{0}=a$ iff $k_{0}=A$

2. $\theta_{0}=b$ iff $k_{0}=B$

3. $\theta_{0}=c$ iff $k_{0}=C$

4. $\theta_{0}=d$ iff $k_{0}=D$

5. $\theta_{0} \in\{a, b, c\}$

Using the doxastic consistency property, $\mathrm{Cr}^{\Theta}$ induces a credibility function $\mathrm{Cr}^{\Omega}[C B]$ about $k_{0}$ given by:

$$
\begin{aligned}
C r^{\Omega}[C B](A) & =C r^{\Theta}(a), \\
C r^{\Omega}[C B](B) & =C r^{\Theta}(b), \\
C r^{\Omega}[C B](C) & =C r^{\Theta}(c), \\
C r^{\Omega}[C B](A, B) & =C r^{\Theta}(a, b), \\
C r^{\Omega}[C B](A, C) & =C r^{\Theta}(a, c), \\
C r^{\Omega}[C B](B, C) & =C r^{\Theta}(b, c), \\
C r^{\Omega}[C B](A, B, C) & =C r^{\Theta}(a, b, c), \\
C r^{\Omega}[C B](D, \omega) & =C r^{\Omega}[C B](\omega), \quad \forall \omega \subseteq\{A, B, C\}
\end{aligned}
$$


The FT piece of evidence. Now You learn for sure that if the killer was one of $A, B, C$, the killer would manage to create a false track. Cigarettes $a, b$ and $c$ can be recognized because, respectively, they have the letters $X Y, X Z$ and $Y Z$ written on them. The killer will purposely drop a butt that points to the other suspects and surely not to him. For example, if the killer was $A, A$ would have managed to let a butt with $X Z$ or $Y Z$ or $Z$ written on it, the last case corresponding to the case where $A$ has erased the missing letter. In the three cases, the butt does not point to $A$.

What $D$ would do is unknown to You.

Let $W$ be the predicate 'You accept as true that exactly ... is written on the butt', so $W(Z)$ means that 'You accept as true that exactly $Z$ is written on the butt' (and thus nothing more, what means in fact that the butt is either a $b$ or a $c$ butt). This information can be written as:

1. If $k_{0}=A$ then $W(X Z) \vee W(Y Z) \vee W(Z)$

2. If $k_{0}=B$ then $W(X Y) \vee W(Y Z) \vee W(Y)$

3. If $k_{0}=C$ then $W(X Y) \vee W(X Z) \vee W(X)$

We write $W()$ to express that nothing is written on the butt. The proposition $W() \vee W(X) \vee W(Y) \vee W(Z) \vee W(X Y) \ldots$

$\vee W(X Z) \vee W(Y Z)$

is true. So we have:

$\neg(W(X Z) \vee W(Y Z) \vee W(Z))=\ldots$

$W() \vee W(X Y) \vee W(X) \vee W(Y)$

and similarly with the other two consequences.

The three rules can be rewritten as

1. If $W() \vee W(X Y) \vee W(X) \vee W(Y)$ then $k_{0} \in\{B, C, D\}$

2. If $W() \vee W(X Z) \vee W(X) \vee W(Z)$ then $k_{0} \in\{A, C, D\}$

3. If $W() \vee W(Y Z) \vee W(Y) \vee W(Z)$ then $k_{0} \in\{A, B, D\}$

Let this piece of evidence be denoted by FT (from False Track).

The problem is to build $C r^{\Omega}[F T]$ and to find out what is the matrix $H$ that transforms $C r^{\Omega}[C B]$ into $C r^{\Omega}[F T]$. This revision is achieved by a contraction on $C B$ and an expansion by $F T$ [3]. The whole subtlety of the example comes from the fact that both $\mathrm{Cr}^{\Omega}[C B]$ and $C r^{\Omega}[F T]$ can be deduced from $\mathrm{Cr}^{\Theta}$ and that $\mathrm{Cr}^{\Theta}$ is not changed by the pieces of evidence $C B$ or $F T$. It results from the fact that Your beliefs about the cigarette brand is not affected by what it implies on who is the killer.

\subsection{Building the $H$ matrix}

Case 1. Suppose You accept as true that the butt is a $c$ butt, thus $W(Y Z)$. So the antecedent of the third rule in $F T$ is true and You deduce $k_{0} \in\{A, B, D\}$.

In that case, Your belief state is represented by $\operatorname{Cr}^{\Theta}(c)=1$, in which case: 


\begin{tabular}{|c|c|c|c|c|c|c|c|}
\hline & $A$ & $B$ & $C$ & $A$ & $A$ & $B$ & $A$ \\
& & & & $B$ & $C$ & $C$ & $B$ \\
\hline$A$ & 0 & 0 & 0 & 0 & 0 & 0 & 0 \\
$B$ & 0 & 0 & 0 & 0 & 0 & 0 & 0 \\
$C$ & 0 & 0 & 0 & 0 & 0 & 0 & 0 \\
$A, B$ & 0 & 0 & 0 & 0 & 0 & 0 & 0 \\
$A, C$ & 0 & 0 & 0 & 0 & 0 & 0 & 0 \\
$B, C$ & 0 & 0 & 0 & 0 & 0 & 0 & 0 \\
$A, B, C$ & 0 & 0 & 0 & 0 & 0 & 0 & 0 \\
$D$ & 0 & 0 & 0 & 0 & 0 & 0 & 1 \\
$A, D$ & 0 & 0 & 0 & 0 & 0 & 1 & 1 \\
$B, D$ & 0 & 0 & 0 & 0 & 1 & 0 & 1 \\
$C, D$ & 0 & 0 & 0 & 1 & 0 & 0 & 1 \\
$A, B, D$ & 0 & 0 & 1 & 0 & 1 & 1 & 1 \\
$A, C, D$ & 0 & 1 & 0 & 1 & 0 & 1 & 1 \\
$B, C, D$ & 1 & 0 & 0 & 1 & 1 & 0 & 1 \\
$A, B, C, D$ & 1 & 1 & 1 & 1 & 1 & 1 & 1 \\
\hline
\end{tabular}

Table 1: The $H$ matrix to transform $C r^{\Omega}[C B]$ into $C r^{\Omega}[F T]$.

- before revision on $F T$, Your beliefs were represented by

$$
\begin{aligned}
C r^{\Omega}[C B, W(Y Z)](\omega) & =1 \text { if } C \in \omega \\
& =0 \text { otherwise }
\end{aligned}
$$

which Möbius transform is given by

$$
\begin{aligned}
m^{\Omega}[C B, W(Y Z)](\omega) & =1 \text { if } \omega=C \\
& =0 \text { otherwise. }
\end{aligned}
$$

- after revision on $F T$, it becomes

$$
\begin{aligned}
C r^{\Omega}[F T, W(Y Z)](\omega) & =1 \text { if }\{A, B, D\} \in \omega \\
& =0 \text { otherwise }
\end{aligned}
$$

In that case the coefficients of the $\{C\}$ column of $H$ satisfy

$$
\begin{aligned}
h(\omega,\{C\}) & =1 \text { if }\{A, B, D\} \subseteq \omega \\
& =0 \text { otherwise }
\end{aligned}
$$

The same holds up to a permutation with $a$ and $b$ (see Table 1).

Case 2. Suppose You are sure that $Z$ is written on the butt, but You could not recognize the other letter. So You accept $W(Z)$ as true. It means in fact that You are sure the butt is a $b$ or a $c$ butt and You have no idea to decide if it is a $b$ or if it is a $c$. So the antecedents of the 
second and third rules in $F T$ are satisfied and You deduce $k_{0} \in\{A, C, D\} \cap\{A, B, D\}=$ $\{A, D\}$

In that case, Your belief state on $\Theta$ is represented by $C r^{\Theta}(\theta)=1$ if $\{b, c\} \subseteq \theta$, and 0 otherwise. (see proposition 2.2) in which case:

- before revision on $F T$, the unique non mull mass of the Möbius transform of Your beliefs $C r^{\Omega}[C B, W(Z)]$ was $m^{\Omega}[C B, W(Z)](B, C)=1$

- after revision on $F T$, Your beliefs become: $\operatorname{Cr}^{\Omega}[F T, W(Z)](\omega)=1$ if $\{A, D\} \subseteq$ $\omega$ and 0 otherwise.

In that case the coefficients of the $\{B, C\}$ column of $H$ satisfy

$$
\begin{aligned}
h(\omega,\{B, C\}) & =1 \text { if }\{A, D\} \subseteq \omega \\
& =0 \text { otherwise. }
\end{aligned}
$$

The same hold up to a permutation with $a$ and $b$ (see Table 1).

Case 3. Suppose You are sure that something is written on the butt, but You could not recognize any letter. It means in fact that You are sure the butt is a $a$ or a $b$ or a $c$ butt and nothing more. So you accept $\mathrm{W}()$ as true. So the antecedents of the three rules in $F T$ are satisfied and You deduce $k_{0} \in\{A, C, D\} \cap\{A, B, D\} \cap\{B, C, D\}=\{D\}$ This implies that $C r^{\Theta}(\theta)=1$ if $\theta=\Theta$ and 0 otherwise, in which case:

- before revision on $F T$, the unique non mull mass of the Möbius transform of Your beliefs $C r^{\Omega}[C B, W()]$ was $m^{\Omega}[C B, W()](A, B, C)=1$

- after revision on FT, Your beliefs become: $\operatorname{Cr}^{\Omega}[F T, W()](\omega)=1$ if $\{D\} \subseteq \omega$ and 0 otherwise.

In that case the coefficients of the $\{A, B, C\}$ column of $H$ satisfy

$$
\begin{aligned}
h(\omega,\{A, B, C\}) & =1 \text { if }\{D\} \subseteq \omega \\
& =0 \text { otherwise. }
\end{aligned}
$$

The nature of the columns of $H$ for those $\omega$ that contain $D$ is not required here as $m^{\Omega}\left[C G, m^{\Theta}\right](\omega)=0$ for all $\omega$ such that $D \in \omega$.

\subsection{General belief on $\Theta$}

In general, Your belief on $\Theta$ is represented by a credibility function $C r^{\Theta}$ with $m^{\Theta}$ being its Möbius transform. Under $C B$, this credibility function on $\Theta$ induced a credibility function $C r^{\Omega}\left[C B, C r^{\Theta}\right]$ on $\Omega$. Its Möbius transform is given by: $m^{\Omega}\left[C B, C r^{\Theta}\right](\omega)=$ $m^{\Theta}(\theta)$ where $\omega$ contains the same letters as $\theta$, but capitalized, and $m^{\Omega}\left[C B, C r^{\Theta}\right](\omega)=0$ if $D \in \omega$.

Suppose $m^{\Theta}(\theta)<0$ for some $\theta \subseteq \Theta$. We consider only two cases: either $\theta=\Theta$ or $\theta$ satisfies $|\theta|=|\Theta|-1$.

Suppose $m^{\Theta}(\Theta)$ is negative. Then $m^{\Omega}\left[C B, C r^{\Theta}\right](\{A, B, C\})=m^{\Theta}(\Theta)<0$. Apply the $H$ transform. That negative mass is allocated to the belief given under $F T$ to 
$D$, and no other mass is given to $D$, so $C r^{\Omega}\left[F T, C r^{\Theta}\right](D)<0$ what violates the non negativity of the credibility functions. Hence $m^{\Theta}(\Theta)$ may not be negative.

Suppose $\theta=\{a, b\}$ and $m^{\Theta}(\{a, b\})$ is negative. Then $m^{\Omega}\left[C B, C r^{\Theta}\right](\{A, B\})=$ $m^{\Theta}(\{a, b\})<0$. Apply the $H$ transform. We have

$$
C r^{\Omega}\left[F T, C r^{\Theta}\right](D)=m^{\Omega}\left[C B, C r^{\Theta}\right](\{A, B, C\}) \geq 0
$$

as just shown and

$$
C r^{\Omega}\left[F T, C r^{\Theta}\right](\{C, D\})=m^{\Omega}\left[C B, C r^{\Theta}\right](\{A, B, C\})+m^{\Omega}\left[C B, C r^{\Theta}\right](\{A, B\}) .
$$

The negativity of $m^{\Omega}\left[C B, C r^{\Theta}\right](\{A, B\})$ implies that $C r^{\Omega}\left[F T, C r^{\Theta}\right](\{C, D\}) \leq C r^{\Omega}\left[F T, C r^{\Theta}\right](\{D\})$ what violates the monotony to inclusion requirement. Hence $m^{\Theta}(\{a, b\})$ may not be negative.

\section{Generalization to any $\Omega$}

The previous example can be extended to any set $\Theta$. It is just a matter of rephrasing it accordingly by multiplying the number of cigarette brands and of suspects. So what it proves is that for any $\Theta, m^{\Theta}(\theta) \geq 0$ whenever $|\theta| \geq|\Theta|-1$.

To prove that credibility functions are belief functions, all we need is to show that if a Möbius mass of the credibility function is negative, we can generate an example where such a credibility function would induce another credibility function where some values are negative.

Suppose a credibility function $C r^{\Omega}$ with $m^{\Omega}$ its Möbius masses. Suppose that for $\omega \subseteq \Omega, m^{\Omega}(\omega)<0$. Three cases must be considered. Either $\omega=\Omega$ or $\omega \subset \Omega, \omega \neq \emptyset$ or $\omega=\emptyset$.

Case $\omega=\emptyset$. In that case, $\operatorname{Cr}^{\Omega}(\Omega)>1$ contrary to the boundness requirement.

Case $\omega=\Omega$. We already know that this case is not acceptable.

Case $\omega \subset \Omega, \omega \neq \emptyset$. Build a coarsening $\Omega^{*}$ of $\Omega$ such that the elements of $\bar{\omega}$ are mapped into one singleton of $\Omega^{*}$, the others being mapped into themselves. Then $m^{\Omega^{*}}(\omega)=$ $m^{\Omega}(\omega)$ is negative. Thus we have build a credibility function on $\Omega^{*}$ that allocates a negative mass to a subset of $\Omega^{*}$ such that $|\omega|=\left|\Omega^{*}\right|-1$. We know that this case is not acceptable.

Therefore the Möbius mass of any credibility function must be non negative, thus credibility functions are belief functions.

\subsection{The Negation of a belief function}

Dubois and Prade [2] have introduced the concept of the negation of a belief function. The basic belief assignment of the negation of a belief function is obtained by transferring the basic belief mass $m^{\Omega}(\omega)$ to $m^{\Omega}(\bar{\omega})$ for all $\omega \subseteq \Omega$. In [9], we have shown that this transformation fits with the idea of a source of evidence that is an absolute liar. Our present example illustrates how to generate such a belief function. 


\section{Conclusions}

We show that if revision of any measure representing quantified beliefs can be represented by a matrix multiplication of the initial beliefs, then the Möbius mass related to the measure must be non negative. This implies that any measure representing quantified beliefs is a belief function. The challenge was to produce an example that produces this effect. Our Mischievous Killer story provides such an example. During this derivation we also illustrate how one can produce the negation of a belief function.

\section{References}

[1] Choquet, G. Theory of capacities. Annales de l'Institut Fourier, Université de Grenoble 5 (1953), 131-296.

[2] Dubois, D., ANd Prade, H. A set-theoretic view of belief functions: logical operations and approximations by fuzzy sets. International Journal of General Systems 12 (1986), 193-226.

[3] Gärdenfors, P. Knowledge in Flux: Modelling the Dynamics of Epistemic States. MIT Press, Cambridge, Mass, 1988.

[4] Kohlas, J., And Monney, P. A. Theory of evidence: A survey of its mathematical foundations, applications and computations. ZOR-Mathematical Methods of Operatioanl Research 39 (1994), 35-68.

[5] Kyburg, JR., H. E. Probability and the Logic of Rational Belief. Wesleyan Univ. Press, 1961.

[6] LEVI, I. The Enterprise of Knowledge. MIT Press, Cambridge, Mass, 1980.

[7] Shafer, G. A Mathematical Theory of Evidence. Princeton Univ. Press. Princeton, NJ, 1976.

[8] SMETs, P. Quantifying beliefs by belief functions: an axiomatic justification. In Proceedings of the 13th International Joint Conference on Artificial Intelligence (San Mateo, CA, 1993), Morgan Kaufmann, pp. 598-603.

[9] Smets, P. The alpha-junctions: combination operators applicable to belief functions. In Qualitative and quantitative practical reasoning, D. Gabbay, R. Kruse, A. Nonnengart, and H. Ohlbach, Eds. Springer, 1997, pp. 131-153.

[10] Smets, P. The normative representation of quantified beliefs by belief functions. Artificial Intelligence 92 (1997), 229-242.

[11] SMETs, P. The transferable belief model for quantified belief representation. In Handbook of Defeasible Reasoning and Uncertainty Management Systems (1998), D. M. Gabbay and P. Smets, Eds., vol. 1, Kluwer, Doordrecht, The Netherlands, pp. 267-301.

[12] Smets, P., And Kennes, R. The transferable belief model. Artificial Intelligence 66 (1994), 191-234. 
[13] VoOrbraAk, F. As Far as I Know: Epistemic Logic and Uncertainty. Dissertation, Utrecht University, 1993.

[14] Walley, P. Statistical Reasoning with Imprecise Probabilities. Chapman and Hall, London, 1991.

[15] Wong, S. K. M., Yao, Y. Y., Bollmann, P., And Brger, H. C. Axiomatization of qualitative belief structure. IEEE Trans. SMC 21 (1990), 726-734. 